\title{
Predictive and Prognostic Molecular Factors in Diffuse Large B-Cell Lymphomas
}

\author{
Stefano A. Pileri ${ }^{1, *(\mathbb{D}}$, Claudio Tripodo ${ }^{2,3}{ }^{,}$Federica Melle ${ }^{1}\left(\mathbb{D}\right.$, Giovanna Motta ${ }^{1}$, Valentina Tabanelli ${ }^{1}$, \\ Stefano Fiori ${ }^{1}$, Maria Carmela Vegliante ${ }^{4}$, Saveria Mazzara ${ }^{1}$, Sabino Ciavarella ${ }^{4}$ and Enrico Derenzini ${ }^{5,6}$ \\ 1 Division of Haematopathology, European Institute of Oncology, IEO IRCCS, Via Ripamonti 435, \\ 20141 Milan, Italy; federica.melle@ieo.it (F.M.); giovanna.motta@ieo.it (G.M.); valentina.tabanelli@ieo.it (V.T.); \\ stefano.fiori@ieo.it (S.F.); saveria.mazzara@ieo.it (S.M.) \\ 2 Tumor Immunology Unit, University of Palermo, 90133 Palermo, Italy; claudio.tripodo@unipa.it \\ 3 Tumor and Microenvironment Histopathology Unit, IFOM, the FIRC Institute of Molecular Oncology, \\ 20139 Milan, Italy \\ 4 Hematology and Cell Therapy Unit, IRCCS-Istituto Tumori 'Giovanni Paolo II', Viale Flacco 65, \\ 70124 Bari, Italy; mc.vegliante@oncologico.bari.it (M.C.V.); s.ciavarella@oncologico.bari.it (S.C.) \\ 5 Division of Haemato-Oncology, European Institute of Oncology, IEO IRCCS, Via Ripamonti 435, \\ 20141 Milan, Italy; enrico.derenzini@ieo.it \\ 6 Department of Health Sciences, University of Milan, Via di Rudinì 8, 20146 Milan, Italy \\ * Correspondence: stefano.pileri@ieo.it or stefano.pileri@unibo.it
}

Citation: Pileri, S.A.; Tripodo, C.; Melle, F.; Motta, G.; Tabanelli, V.; Fiori, S.; Vegliante, M.C.; Mazzara, S.; Ciavarella, S.; Derenzini, E. Predictive and Prognostic Molecular Factors in Diffuse Large B-Cell Lymphomas. Cells 2021, 10, 675. https://doi.org/ 10.3390/cells10030675

Academic Editor: Caterina Marchiò

Received: 12 January 2021

Accepted: 12 March 2021

Published: 18 March 2021

Publisher's Note: MDPI stays neutral with regard to jurisdictional claims in published maps and institutional affiliations.

\begin{abstract}
Diffuse large B-cell lymphoma (DLBCL) is the commonest form of lymphoid malignancy, with a prevalence of about $40 \%$ worldwide. Its classification encompasses a common form, also termed as "not otherwise specified" (NOS), and a series of variants, which are rare and at least in part related to viral agents. Over the last two decades, DLBCL-NOS, which accounts for more than $80 \%$ of the neoplasms included in the DLBCL chapter, has been the object of an increasing number of molecular studies which have led to the identification of prognostic/predictive factors that are increasingly entering daily practice. In this review, the main achievements obtained by gene expression profiling (with respect to both neoplastic cells and the microenvironment) and next-generation sequencing will be discussed and compared. Only the amalgamation of molecular attributes will lead to the achievement of the long-term goal of using tailored therapies and possibly chemotherapy-free protocols capable of curing most (if not all) patients with minimal or no toxic effects.
\end{abstract}

Keywords: diffuse large B-cell lymphoma; gene expression profiling; next-generation sequencing; classification; diagnosis; prognosis; therapy

\section{Classification}

Diffuse large B-cell lymphoma (DLBCL) is the most common form of lymphoid malignancy, with a prevalence of about $40 \%$ worldwide [1]. It consists of medium or large B-lymphoid cells in which the nuclei are the same size as or larger than those of normal macrophages, or more than twice the size of those of normal lymphocytes, with a diffuse growth pattern [1]. The concept of DLBCL has undergone fine-tuning over time, as is clear from the comparisons between the REAL and WHO classifications (third, fourth, and revised fourth editions) [1-4]. This produces some apparent terminological discrepancies throughout the text, which reflect the time of publication of each reference.

In the Revised Fourth Edition of the WHO Classification of Tumours of Haematopoietic and Lymphoid Tissues, DLBCL is subdivided into morphologic variants, molecular subtypes, and distinct disease entities (Table 1) [1]. Nevertheless, about 70\% of all DLBCLs lack features allowing their inclusion into one of the diagnostic categories listed in Table 1 [1]. These cases are collectively termed as "not otherwise specified" (DLBCL-NOS) [1] and are conventionally treated with the chemoimmunotherapy regimen $\mathrm{R}-\mathrm{CHOP}[5,6]$. 
Table 1. Diffuse large B-cell lymphoma, high-grade B-cell lymphoma, and gray-zone lymphoma according to the Revised Fourth Edition of the WHO Classification of Tumours of Haematopoietic and Lymphoid Tissues (italics indicate that an entity is provisional).

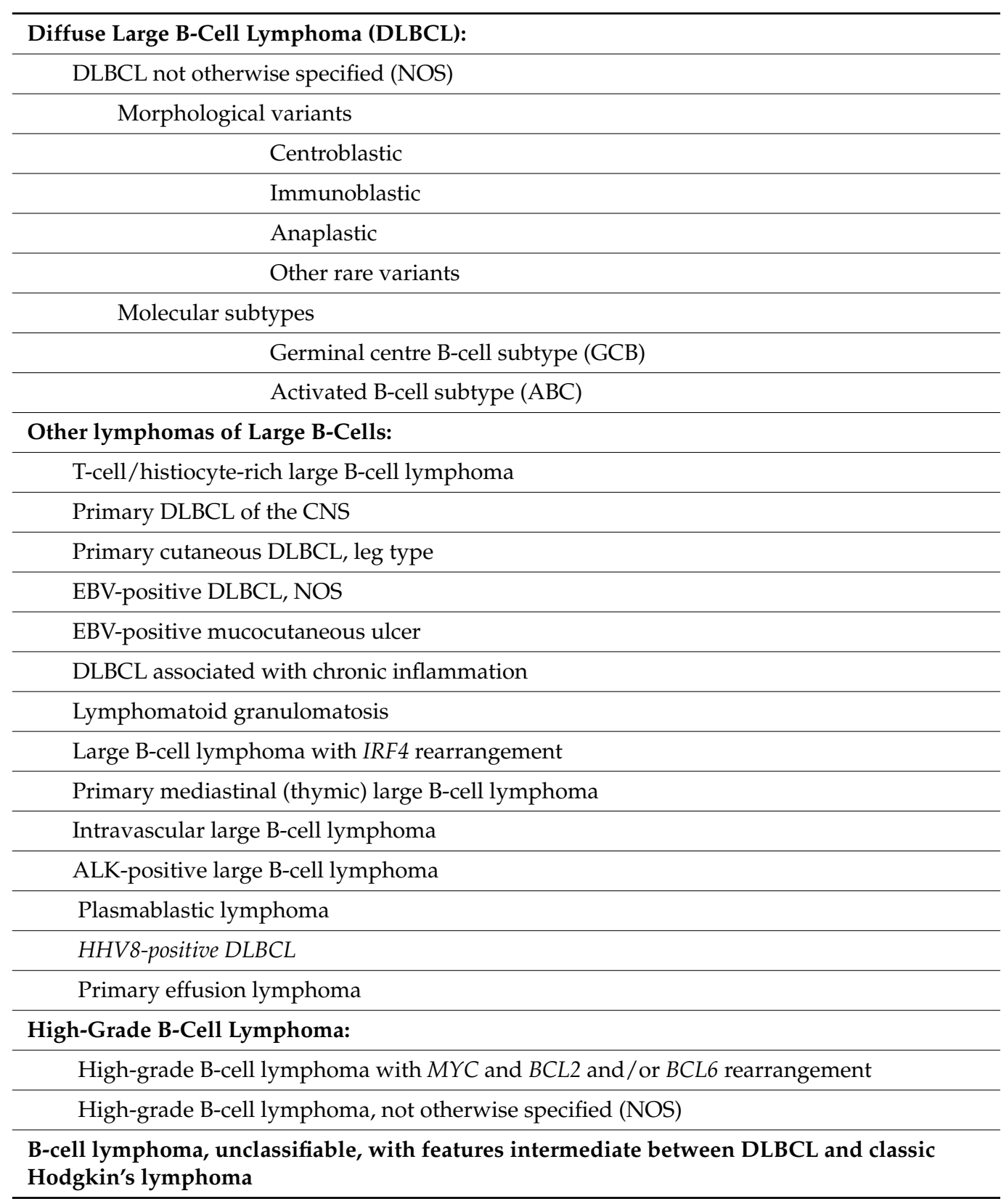

Based on a recent survey of 3550 DLBCL patients who mostly underwent R-CHOP with curative intent, the 5-year overall survival and cumulative incidence of relapsed/refractory disease corresponds to $65.3 \%$ and $23.1 \%$ of cases, respectively [6]. Thus, there is still an unmet need for optimal therapy for a significant proportion of DLBCL-NOS patients.

In recent years, DLBCL-NOS has been the object of the extensive application of highthroughput technologies, which has led to the identification of prognostic/predictive factors that are increasingly entering daily practice.

Although DLBCL-NOS is the main focus of this review, the borders between DLBCLNOS and high-grade B-cell lymphoma (HGBCL) (Table 1) will also be discussed. In fact, it is not uncommon to encounter cases that could be regarded as DLBCL-NOS but are ultimately classified as HGBCL due to the detection of double or triple hits $(\mathrm{D} / \mathrm{TH})$ of MYC, BCL2, and/or BCL6 (HGBCL-D/TH) by FISH, as underlined by Sehn and Salles 
in their review on DLBCL published in the New England Journal of Medicine on 4 March 2021 [7] (see below).

\section{Gene Expression Profiling}

2.1. Cell of Origin (COO)

At the beginning of this century, using gene expression profiling (GEP) Alizadeh and coworkers first reported that DLBCLs could be divided into two main subtypes with a gene signature related to the germinal center B-cell (GCB) and activated B-lymphocytes from the peripheral blood (ABC), respectively [8]. Such a distinction, not feasible on morphological grounds, had an important prognostic impact. In fact, the GCB forms had a significantly more favorable response to chemotherapy (CHOP) than those of ABC. This corresponded to a clear-cut difference in terms of overall and progression-free survival (OS and PFS, respectively). This subdivision was subsequently confirmed using cohorts consisting of hundreds of cases, and maintained its value in the era of chemoimmunotherapy [9-11]. By expanding the number of profiled cases, a third group between those of GCB and $A B C$ emerged and was indicated as unclassified (U), corresponding to about $15 \%$ of DLBCLs [9-11]. Besides prognostic value, the distinction between GCB and ABC subtypes has biological relevance as it corresponds to different genetic aberrations as well as pathway perturbations (as detailed in the following).

The main limitation of conventional GEP was the need for fresh or frozen (FF) samples, which were available for a small minority of patients followed up at reference centers. Therefore, many attempts were made to find surrogates for GEP through the search for immunohistochemical markers [12-18]. Several algorithms were proposed, with that of Hans et al. having the widest applications as it was based on the simple determination of CD10, BCL6, and IRF4/MUM1 [12]. However, none of these algorithms met their goal, for several reasons: (a) a lack of correspondence with GEP data in the same patients; (b) variability in the preanalytical and immunohistochemical techniques (including antibody and antigen retrieval, detection systems, and automatic platforms); and (c) subjectivity in result interpretation $[19,20]$.

In 2014, a new approach was proposed based on targeted digital GEPFF and was successfully applied to mRNA extracted from formalin-fixed, paraffin-embedded (FFPE) tissue samples (Lymph2Cx) [21]. In particular, a 20-gene panel (including 15 top genes and 5 housekeeping genes for normalization) was designed, which in 67 cases provided the same COO classification as conventional GEP from FF. Furthermore, the OS and PFS curves were over-imposable, irrespective of the type of GEP used (targeted digital vs. conventional). These preliminary results, which had been obtained by using the NanoString platform, were subsequently confirmed by independent studies based on several hundred cases [22-25]. The advantages of this approach over immunohistochemical algorithms are: (1) reproducibility in different laboratories; (2) the assessment of the absolute value of mRNA expressed by each gene; and (3) a lack of confounding factors (such as the variability of immunohistochemical techniques and subjective result interpretation). Moreover, targeted GEP subdivides DLBCLs-NOS into GCB, ABC, and U, like conventional profiling of FF samples. In contrast, immunohistochemical algorithms differentiate DLBCLs-NOS into GCB and non-GCB, with the latter group containing cases that are molecularly classified as GCB [21-25]. Interestingly, identical results were obtained by targeted profiling on different platforms and with different panels of genes confirming the prognostic relevance of the COO determination [21-25].

The COO determination provided less significant prognostic information when applied to cases enrolled in some trials [26-28]. This may be for several different reasons, i.e., (1) the adoption of protocols that are more intense than those used in real situations; (2) the selection of patients fit enough to await the completion of all the tests required for trial enrolment; and (3) the influence of other factors that can affect behavior within each subgroup defined by the $\mathrm{COO}$. 
The main limitation of targeted GEP applied to routine biopsies is the need for platforms which are not available in all pathology laboratories, unlike immunohistochemistry. This problem, as well as the test costs and need for basic bioinformatic skills, can be overcome by a hub-and-spoke organization, which is also required for the application of the array of molecular techniques at the basis of precision medicine (see below).

\subsection{Key Genes}

FISH analyses have shown that B-cell lymphomas, regarded as DLBCLs-NOS based on morphology and phenotype, could carry double or triple rearrangements of $M Y C, B C L 2$, and/or BCL6 [1]. These cases, which overall have a significantly worse prognosis and may require therapies that are more intense than standard $\mathrm{R}-\mathrm{CHOP}$, are nowadays included in the provisional category of high-grade B-cell lymphomas with double/triple hits (HGBL $\mathrm{D} / \mathrm{TH}$ ). Based on this observation, FISH should ideally be applied to all DLBCL-NOS cases. As FISH analyses are rather expensive, attempts have been made to find surrogates for FISH results through immunohistochemistry. This has led to the identification of a group of DLBCL-NOS cases which show double expression of MYC and BCL2 at the protein level (the so-called double expressors (DEs)) [29,30]. According to the Revised Fourth Edition of the WHO Classification of Tumours of Haematopoietic and Lymphoid Tissues, at least $50 \%$ and $40 \%$ of neoplastic cells should express BCL2 and MYC, respectively, in order to consider a DLBCL-NOS patient as a DE [1]. However, discrepancies exist as to the reproducibility of the cut-off value of MYC positivity, which has been moved to $70 \%$ by some groups [31]. Most importantly, as there is no actual correspondence between the results of immunohistochemistry and FISH [32], these cases with MYC and BCL2 doubleexpression but lacking D/TH remain within the bounds of DLBCL-NOS but more often belong to the $\mathrm{ABC} /$ non-GCB subtype and require further studies to definitively assess their prognostic and/or therapeutic relevance $[1,32]$.

In 2018, investigators from two groups used GEP signatures to identify high-risk patients with DLBCL in FFPE series. Sha et al. [33] used a Burkitt lymphoma-like signature, whereas Ennishi et al. [34] used a signature derived from genes differentially expressed between $M Y C / B C L 2 \mathrm{DH}$ and non-DH GCB-DLCBLs. With their respective signatures, these investigators were, as expected, able to identify most DH lymphomas, as well as many non-DH lymphomas which were actually found in about half of the identified patients and showed a poorer response to standard chemoimmunotherapy. These findings suggest that many of the patients harbored genetic or even epigenetic alterations that produced similar gene expression changes in the tumor cells, as recognized by the respective signatures. This does not come as a surprise. In fact, activation of an oncogene or oncogenic pathway can be produced by multiple mechanisms besides MYC/BCL2 DH, for example $M Y C$ upregulation through translocation and gene amplification. Apart from structural alterations, MYC expression or activity can be enhanced through transcriptional and posttranscriptional events.

Derenzini et al. [25] used a targeted GEP panel combining the Lymph2Cx signature for COO classification, with additional targets including MYC, BCL-2, and NFKBIA (the latter encoding for the IkB- $\alpha$ protein, an endogenous inhibitor of NF-kB signaling [35]), in 186 FFPE cases originally diagnosed as DLBCL-NOS from two randomized trials (discovery cohorts NCT00355199 and NCT00499018) and in three independent validation cohorts. By integrating the $\mathrm{COO}, M Y C / B C L-2$ DE status, and NFKBIA expression, a three-gene signature was designed combining $M Y C, B C L-2$, and NFKBIA (MBN signature). The high-risk (MBN Sig-high) subgroup, characterized by higher expression levels of $M Y C$ and $B C L-2$ and a lower expression of NFKBIA, could be used to identify a significant fraction of ABC DLBCLs and the vast majority of double-hit cases, allowing for further risk stratification within the $\mathrm{GCB} / \mathrm{U}$ subset. These results were validated in three independent series including Sha's cohort based on the REMoDL-B trial $[33,36]$, a phase III randomized trial investigating the efficacy of the addition of bortezomib to standard first-line chemoimmunotherapy. In line with the biological activity of bortezomib, which increases the protein abundance of IkB- $\alpha$, leading to inhibition of NF-kB signaling [37], an exploratory ad hoc analysis of the latter cohort showed that the addition of bortezomib in the MBN Sig-high subgroup provided 
a progression-free survival advantage compared with standard chemoimmunotherapy. These data suggest that a simple three-gene signature based on MYC, BCL-2, and NFKBIA can refine the prognostic stratification in DLBCL.

Finally, Mottok et al. [38] developed a robust and accurate molecular classification assay (Lymph3Cx) for the distinction of primary mediatinal B-cell lymphoma (PMBCL) from DLBCL subtypes based on gene expression measurements in formalin-fixed, paraffinembedded tissue. A probabilistic model accounting for classification error comprising 58 gene features was trained on 68 cases of PMBCL and DLBCL. Model performance was subsequently evaluated in an independent validation cohort of 158 cases and showed a high agreement of the Lymph3Cx molecular classification with the clinicopathological diagnosis of an expert panel (frank misclassification rate, 3.8\%). In the authors' view, Lymph3Cx represents a molecular tool that is potentially helpful for the diagnosis of PMBCL in light of the use of ad hoc therapeutic approaches [1]. In fact, on central review, cases enrolled in trials as DLBCL-NOS are not infrequently reclassified as PMBCL and vice versa, as seen in the authors' experience at a reference center for several trials of the Italian Lymphoma Foundation.

\section{Tissue Microenvironment (TME)}

By means of a gene profiling analysis of nearly 500 FF DLBCL samples, in 2008 Lenz et al. first demonstrated that the expression of peculiar gene sets, namely "Stromal-1" and "Stromal-2" signatures, respectively correlated with good- and poor-outcome subgroups of R-CHOP-treated patients independently of COO [39]. Although they were selectively enriched in genes encoding extracellular matrix proteins and histiocyte infiltration (Stromal-1) or reflecting angiogenesis (Stromal-2), these signatures resulted mechanistically uninformative, and their practical use was limited by the lack of standardized GEP assays for FFPE samples. A number of subsequent research attempts were aimed at identifying TME-related prognostic factors, but none provided biomarkers reproducible enough to be translated into daily clinical practice [40-43].

To overcome this limitation, in 2018 Ciavarella and co-workers [44] generated a 1028-gene matrix incorporating the signatures of 17 cytotypes and applied the computational method CIBERSORT to deconvolve Lenz's GEP dataset. The work clarified the prognostic associations between patient outcome and quantitative proportions of tumor-infiltrating cell types. A panel of 45 genes related to myofibroblasts (MFs), dendritic cells, and CD4+ T-cells was selected and digitally validated by a NanoString-based approach on an independent cohort of 175 FFPE DLBCLs from two randomized trials. All tissue samples consisted of pretreatment biopsies of advanced-stage nodal DLBCLs treated by comparable R-CHOP/R$\mathrm{CHOP}$-like regimens. The expression of the $45 \mathrm{TME}$ genes positively correlated with better outcomes and predicted the patient risk of overall and progression-free survival. In a multivariate Cox model, the TME panel retained high prognostic performance independently of $\mathrm{COO}$, and integration of the two prognostic factors (COO + TME) improved survival prediction. Finally, a model to assign single DLBCL cases to a "COO-TME" risk category was built and successfully applied to an independent cohort of 40 "real-life" cases.

In a parallel work, Staiger et al. [45] proposed a lymphoma-associated macrophage interaction signature (LAMIS) interrogating features of the microenvironment, once again using a NanoString assay applicable to FFPE. The clinical impact of the signature was validated in a cohort of 466 patients enrolled in prospective clinical trials at the German HighGrade Non-Hodgkin Lymphoma Study Group (DSHNHL). Patients with high expression of the signature (LAMIS-high) had shorter event-free survival (EFS), progression-free survival (PFS), and overall survival (OS). Multivariate analyses revealed independence from International Prognostic Index (IPI) factors in EFS (HR 1.7, 95\%CI 1.2-2.4, $p$-value $=0.001$ ), PFS $($ HR 1.8, 95\%CI 1.2-2.5, $p$-value $=0.001)$, and OS (HR 1.8, 95\%CI 1.3-2.7, $p$-value $=0.001)$. Multivariate analyses adjusted for the IPI factors showed the signature was independent of COO, MYC rearrangements, and double-expressor (DE) status. LAMIS-high and simultaneous DE status characterized a patient subgroup with dismal prognosis and greater probability of early relapse. 
Beyond the prognostic value of TME, only a few studies have provided comprehensive biological insights into the putative link between B-cell genomics and functional patterns of immune and stromal components while suggesting new rationales for future therapeutic approaches.

Tripodo et al. [46] reported on a spatially resolved 53-gene signature comprising key genes of the dark-zone (DZ) mutational machinery, and light-zone (LZ) immune and mesenchymal milieu. This signature was applied to the transcriptomes of 543 cases of GCBDLBCL and HGBCL-DH. According to the DZ/LZ signature, the GC-related lymphomas were sub-classified into two clusters. The subgroups differed in the distribution of $\mathrm{DH}$ cases and survival, with most DH cases displaying a distinct DZ-like profile. The clustering analysis was also performed using a 25-gene signature composed of DZ/LZ genes positively enriched in the non-B, stromal sub-compartments, for the first time achieving DZ/LZ discrimination based on stromal/immune features. The report offers new insight into the GC microenvironment, hinting at a DZ microenvironment of origin in DH lymphomas.

Intriguing research integrating transcriptomic, genetic, and immunophenotypic data of 347 DLBCLs demonstrated that MHC loss, particularly in GC-derived tumors originating from the centroblast-rich DZ, is associated with a strong enrichment of EZH2 mutations, lower $T$ cell infiltration, and poorer outcome [47]. Such results paved the way for the potential use of EZH2 inhibitors to treat the tumor by simultaneously modulating its immune microenvironment.

Finally, very recent work by Kotlov et al. [48] provided a relevant classification of DLBCL based on the transcriptomic characterization of TME from 4655 cases. Four major TME categories were identified as being associated with peculiar genetic/epigenetic aberrations of the malignant component, clinical behavior, and potential therapeutic targeting. Beyond its classification value, to date this work represents the most extensive translational and biological analysis of malignant and non-malignant DLBCL components.

By all means, in-depth TME analysis still represents an approach that can significantly improve the prognostication of DLBCL and even further tune the identification of different risk groups within the same $\mathrm{COO}$ category, predicting the response to targeted therapies.

\section{Genetic Classification}

Over the last few years, several proposals for a genetic classification of DLBCL have been published. Hereunder, the main contributions will be summarized and discussed based on the technical approach used.

\subsection{Whole-Exome Sequencing (WES)-Based Studies}

In 2017, Reddy and coworkers [49] reported on the whole-exome sequencing (WES) of 1001 FF DLBCLs and 400 paired germline DNAs. They found 150 driver genes to be recurrently mutated. The 60 top genes frequently exhibited a pattern of either predominant missense and/or copy number gains consistent with an oncogene or truncating mutations and/or copy number losses consistent with a tumor suppressor gene. When the mutational pattern was matched with the $\mathrm{COO}, 20$ genes were differentially mutated between the two groups, including EZH2, SGK1, GNA13, SOCS1, STAT6, and TNFRSF14, which were mutated in GCB tumors, and ETV6, MYD88, PIM1 and TBL1XR1, which were mutated in $A B C$ tumors. Interestingly, MLL2 mutations were associated with those of $M Y C$, while TP53 mutations occurred in a mutually exclusive fashion with KLHL6. CRISPR screening revealed that knockout of EBF1, IRF4, CARD11, MYD88, and IKBKB was selectively lethal in ABC DLBCL cell lines, as was knockout of ZBTB7A, XPO1, TGFBR2, and PTPN6 in the GCB lines. On prognostic grounds, $M Y C$ mutations were strongly associated with poorer survival, as were mutations in CD79B and ZFAT. Mutations in NF1 and SGK1 were associated with more favorable survival. Furthermore, in ABC DLBCLs, genetic alterations in KLHL14, BTG1, PAX5, and CDKN2A were associated with significantly poorer survival, while those in CREBBP were associated with favorable outcomes. In the GCB-DLBCL group, genetic alterations in NFKBIA and NCOR1 were associated with poorer prognosis, while alterations in $E Z H 2, M Y D 88$, and $A R I D 5 B$ were all associated with a significantly better prognosis. The authors developed a multivariate supervised learning approach for 
defining the association of survival with combinations of genetic markers (150 genetic driver genes) and gene expression markers (cell of origin, MYC, and BCL2). This led to the proposal of a three-subgroup molecular risk model that was found to outperform all existing predictors (i.e., COO, MYC/BCL2 DE, and IPI). However, the recent application of this model to 499 DLBCLs by Bolen et al. [50] did not provide independent validation. This might reflect the technical differences between the two studies (WES of FF samples by Reddy et al. vs. targeted NGS of DNA extracted from FFPE biopsies by Bolen et al.).

Two studies published in 2018 proposed a molecular subclassification of DLBCLs that had potential prognostic and therapeutic implications [51,52]. They both were based on WES and copy-number analysis of a large series of FF DLBCLs (304 and 574, respectively) [51,52].

Chapuy et al. [51] described five clusters characterized by different genetic lesions that were capable of identifying subgroups within the $\mathrm{COO}$ categories showing different behaviors.

Most cases included in clusters (Cs) 1 and 5 were classified as ABC. However, they showed important differences on molecular and prognostic grounds. $\mathrm{C} 1$ cases were thought to derive from marginal-zone B-cells, as they showed a stable mutational pattern, structural variants (SVs) of $B C L 6$, and mutations of genes involved in the NOTCH2 and NF-kB pathways (NOTCH2, SPEN, BCL10, TNFAIP3, and FAS). Besides the multiple genetic lesions of genes involved in immune escape (BM2, CD70, FAS, PD-L1, PD-L2), these C1 cases carried MYD88 mutations which were non-L265P, unlike what was observed in the cases included in C5. Notably, C1 cases had a rather favorable course and revealed potential therapeutic targets related to NOTCH2 and BCL6 signaling and immune evasion mechanisms. $C 5$ tumors, which behaved more aggressively than the $\mathrm{C} 1$ ones, showed mutations of MYD88 L265P , CD79B, PIM1, TBL1XR1, GRHPR, and BTG1, SV of 18q, and activation of the NF-kB pathway. In addition, they carried ongoing mutations, being at least in part under the effect of AID. Potential targets for C5 cases corresponded to BCR/TLR signaling and BCL2.

Cs 3 and 4 were significantly enriched in GCB cases but were characterized by different genetic lesions and responses to chemoimmunotherapy. The majority of DLBCLs in C3 harbored BCL2 mutations with concordant SVs. They also exhibited frequent mutations in chromatin modifiers, KMT2D, CREBBP, and $E Z H 2$, and increased transcriptional abundance of EZH2 targets by gene set enrichment analisys (GSEA). These tumors also had alterations in the B-cell transcription factors $M E F 2 B$ and IRF8, and indirect modifiers of BCR and PI3K signaling (TNFSF14(HVEM), HCNV1, and GNA13). In addition, C3 tumors had two alternative mechanisms of inactivating PTEN: focal 10q23.31/PTEN loss and predominantly truncating PTEN mutations, events that play a role in the process of lymphomagenesis. C4 DLBCLs were characterized by mutations in four linker and four core histone genes, multiple immune evasion molecules (CD83, CD58, and CD70), BCR/PI3K signaling intermediates (RHOA, GNA13, and SGK1), NF-kB modifiers (CARD11, NFKBIE, and NFKBIA), and RAS/JAK/STAT pathway members (BRAF and STAT3). Comparison of the C3 and C4 genetic signatures further revealed that these GCB-DLBCLs utilized distinct mechanisms to perturb common pathways such as PI3K signaling. In contrast to C3 DLBCLs, C4 tumors rarely exhibited PTEN alterations but harbored more frequent RHOA mutations. In addition, C4 DLBCLs rarely exhibited BCL2 alterations and had higher mutational density. The distinct genetic features of C3 and C4 GCB-DLBCLs led Chapuy et al. to suggest specific targeted therapies including inhibition of $\mathrm{BCL} 2, \mathrm{PI} 3 \mathrm{~K}$, and the epigenetic modifiers EZH2 and CREBBP in C3 GCB tumors, and JAK/STAT and BRAF/MEK1 blockade in C4 GCB-DLBCLs. Last but not least, C3 cases had a far worse prognosis.

C2 DLBCLs harbored frequent biallelic inactivation of TP53 by mutations and 17p copy loss. In addition, they often exhibited copy loss of $9 p 21.13 / C D K N 2 A$ and 13q14.2/RB1, perturbing chromosomal stability and cell cycle. $\mathrm{C} 2$ tumors also had significantly more driver somatic copy number alterations (SCNAs) and a higher proportion of genome doubling events. This cluster included both GCB- and ABC-DLBCLs, as did prior DLBCL cohorts with TP53 mutations in targeted analyses [53]. Prognostically significant SCNAs, including 13q31.31/miR-17-92 copy gain and 1q42.12 copy loss, were also more common in these DLBCLs, which were characterized by a rather unfavorable prognosis. 
A further cluster, termed 0 , was also detected, which apparently lacked significant genetic alterations. However, as the $\mathrm{C} 0$ group consisted almost exclusively of T-cell rich/histiocyte-rich B-cell lymphomas, the obtained results might have been largely influenced by the small number of neoplastic cells.

The authors further evaluated BCL2 and MYC alterations. Tumors with cooccurring $B C L 2$ and MYC SVs were significantly more frequent in C3 DLBCLs.

Importantly, the coordinate genetic signatures reported by Chapuy et al. predicted outcomes independent of IPI which could suggest new combination treatment strategies and, more broadly, provide a roadmap for actionable DLBCL classification [51].

By their integrated approach, Schmitz et al. [52] identified four prominent genetic subtypes among 574 DLBCLs which they termed MCD (based on the co-occurrence of $M Y D 88^{L 265 P}$ and $C D 79 B$ mutations), BN2 (based on BCL6 fusions and NOTCH2 mutations), $\mathrm{N} 1$ (based on NOTCH1 mutations), and EZB (based on EZH2 mutations and BCL2 translocations). Interestingly, Schmitz and co-workers enriched their series with unclassified DLBCLs. The latter turned out to frequently carry mutations affecting SPEN and NOTH2 as well as BCL6 fusions. ABC cases were enriched in MYD $88^{L 265 P}$ and CD79B or NOTCH1 mutations, with the two conditions being mutually exclusive. GCB tumors showed the co-occurrence of $E Z H 2$ mutations and $B C L 2$ translocations. The MCD and N1 subtypes were dominated by ABC cases, while EZB included mostly GCB tumors, and BN2 had contributions from all GEP subgroups. Overall, about $45 \%$ of the samples were classified into the genetically pure subtypes of DLBCL.

The MCD subtype displayed $82 \%$ of cases carrying MYD $88^{\mathrm{L} 265 \mathrm{P}}$ or $C D 79 B$ aberrations (mutation or amplification), with $42 \%$ bearing both abnormalities. The MCD subtype showed a frequent gain or amplification of SPIB, encoding a transcription factor that, with IRF4, defines the $A B C$ phenotype and promotes plasmacytic differentiation. Known tumor suppressors in MCD include CDKN2A, ETV6, BTG1, and BTG2, and putative tumor suppressors include TOX, SETD1B, FOXC1, TBL1XR1, and KLHL14. The tumor suppressor TP53 was mutated significantly less often in MCD as compared to other subtypes. Immune editing appeared prominent in MCD genomes, with $76 \%$ acquiring a mutation or deletion of $H L A-A, H L A-B$, or $H L A-C$ and $30 \%$ acquiring truncating mutations targeting CD58.

BN2 was dominated by NOTCH pathway aberrations, with $73 \%$ acquiring a NOTCH2 mutation or amplification, SPEN mutation, or mutation in DTX1, a NOTCH target gene. $B C L 6$ fusion, the other BN2 hallmark, occurred in $73 \%$ of cases. BCL6 fusions were enriched in cases with NOTCH2, SPEN, or DTX1 lesions to a significantly greater extent in BN2 than in non-BN2 cases. Genetic aberrations (mutations or amplifications) targeting regulators of the NF-kB pathway were a prominent feature of BN2. These more often affected TNFAIP3, $P R K C B$, and BCL10. Other likely gain-of-function events included mutations targeting cyclin $D 3$ and CXCR5, whereas inactivating lesions targeting the immune regulator CD70 suggested immune escape.

N1 was characterized by NOTCH1 mutations and aberrations targeting transcriptional regulators of B-cell differentiation (IRF4, ID3, and BCOR), which may contribute to its plasmacytic phenotype. TNFAIP3 mutations in N1 could reinforce this phenotype by fostering NF-KB-induced IRF4 expression.

EZB was enriched for most of the genetic events previously ascribed to GCB-DLBCL, including $B C L 2$ translocation, EZH2 mutation, and REL amplification, as well as inactivation of the tumor suppressors TNFRSF14, CREBBP, EP300, and KMT2D. The germinalcenter homing pathway involving S1PR2 and GNA1314 was disrupted in 38\% of EZB cases. JAK-STAT signaling was promoted in about half cases by a STAT6 mutation or amplification or by a mutation or deletion targeting SOCS1. PI3K target of rapamycin signaling turned out to be activated in $23 \%$ of cases by MTOR mutations or the amplification of MIR17HG. Immune editing was of interest in EZB genomes since 39\% acquired lesions in the major histocompatibility complex class II pathway genes CIITA and HLA-DMA.

The four subtypes differed significantly in PFS and OS, with the BN2 and EZB subtypes having much more favorable outcomes than the MCD and N1 subtypes. The predicted 5 -year OS rates for the MCD, N1, BN2, and EZB subtypes were $26 \%, 36 \%, 65 \%$, and $68 \%$, respectively. Within $A B C D L B C L$, patients with MCD had significantly inferior survival 
as compared with those with BN2, and patients with either MCD or N1 had significantly inferior survival as compared with patients with $\mathrm{ABC}$ tumors that were not genetically classified. Within GCB-DLBCL, there was a trend toward inferior OS among patients with EZB as compared with those with other GCB tumors. The COO subgroups and genetic subtypes independently contributed to survival in a multivariate analysis. Conversely, the IPI score did not vary significantly among the genetic subtypes, but the latter significantly added to IPI. A trend toward increased extranodal involvement (e.g., CNS) was a feature of MCD, which reflected the frequent $C D 79 B$ and $M Y D 88^{\mathrm{L} 265 \mathrm{P}}$ mutations.

On therapeutic grounds, constitutive BCR signaling activation was most frequent in MCD and least frequent in EZB, but genetic alterations involving the BCR cascade occurred in all genetics subtypes, suggesting that constitutive BCR signaling is a pervasive aspect of DLBCL pathogenesis. BN2 was notably enriched for BCR-NF-kB and IKK regulator aberrations. In addition to NF-kB, survival of DLBCL cells turned out to be promoted by antiapoptotic BCL2 family members, which were targeted by genomic amplification or translocation in $17.4 \%$ of cases. As expected, BCL2 mRNA levels were significantly higher in EZB tumors with BCL2 translocations than in other EZB tumors. MCD tumors also had high BCL2 mRNA expression as compared with other cases, a finding due to mechanisms other than translocation or amplification.

\subsection{Targeted NGS and Bioinformatic-Based Studies}

Lacy et al. [54] applied a 293-gene chip to DNA extracted from FFPE tissue samples by using a Covaris LE220. The authors sequenced a large, unselected cohort consisting of 928 DLBCL patients all treated with R-CHOP and provided with full clinical follow-up. Bernoulli mixture-model clustering was applied, and the resulting subtypes analyzed in relation to their clinical characteristics and outcomes. Five molecular subtypes were resolved, termed MYD88, BCL2, SOCS1/SGK1, TET2/SGK1, and NOTCH2, along with an unclassified group. The subtypes characterized by genetic alterations of BCL2, NOTCH2, and MYD88 recapitulated the above-mentioned studies showing good, intermediate, and poor prognosis, respectively. The SOCS1/SGK1 subtype showed biological overlap with primary mediastinal B-cell lymphoma and conferred excellent prognosis. Although not identified as a distinct cluster, NOTCH1 mutation was associated with poor prognosis. The impact of TP53 mutation varied with genomic subtypes, conferring no effect in the NOTCH2 subtype and poor prognosis in the MYD88 subtype. The results obtained by Lacy et al. are summarized in Table 2, where they are also compared with the subtypes reported by Chapuy et al. [51], and Schmitz et al. [52].

Ennishi et al. [55] performed an integrative genomic and transcriptomic analysis of DLBCL using a British Columbia population-based registry. They uncovered recurrent biallelic TMEM30A loss-of-function mutations which were associated with a favorable outcome and were uniquely observed in DLBCL. Using TMEM30A-knockout systems, increased accumulation of chemotherapy drugs was observed in TMEM30A-knockout cell lines and TMEM30A-mutated primary cells, accounting for the improved treatment outcome. Furthermore, they found increased tumor-associated macrophages and an enhanced effect of anti-CD47 blockade limiting tumor growth in TMEM30A-knockout models. By contrast, Ennishi et al. showed that TMEM30A loss-of-function increased B-cell signaling following antigen stimulation-a mechanism conferring selective advantage during B-cell lymphoma development. These findings suggested intrinsic and extrinsic vulnerabilities of cancer cells that can be therapeutically exploited.

Finally, Wright et al. [56] developed an algorithm to determine the probability of a patient's lymphoma belonging to one of seven genetic subtypes based on its genetic features. This represented a probabilistic classification tool (LymphGen) using any combination of mutational, copy number, and BCL2/BCL6 rearrangement data. Schmitz's cohort was used as training set, while those from Chapuy et al. and Ennishi et al. were used for validation. Wright et al. developed a model that is summarized in Table 3, which also includes information on potential therapeutic targets related to the genetic subtype of DLBCL. 
Table 2. Molecular subtypes of DLBCL according to Lacy et al. in comparison with those of Chapuy et al. and Schmitz et al.

\begin{tabular}{|c|c|c|c|c|}
\hline Lacy et al. & Chapuy et al. & Schmitz et al. & & Notes \\
\hline \multirow{6}{*}{$\sum_{\substack{\infty \\
\infty}}^{\infty}$} & \multirow{6}{*}{ 눈 } & \multirow{6}{*}{$\stackrel{\theta}{\Sigma}$} & MYD88 & \multirow{6}{*}{$\begin{array}{l}\text { Strongly associated with ABC. The most robust } \\
\text { group in all reports. Contains the most primary } \\
\text { PCNSL and testicular lymphoma. Poor prognosis. }\end{array}$} \\
\hline & & & CD79B & \\
\hline & & & PIM1 & \\
\hline & & & ETV6 & \\
\hline & & & CDKN2A & \\
\hline & & & TBL1XR1 & \\
\hline \multirow{7}{*}{$\underset{ٍ}{\mathcal{U}}$} & \multirow{7}{*}{3} & \multirow{7}{*}{ 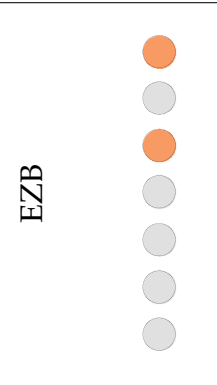 } & $E Z H 2$ & \multirow{7}{*}{$\begin{array}{l}\text { Strongly associated with GCB. Contains most } \\
\text { transformed FLs and cases with a concurrent FL. } \\
\text { Generally favorable prognosis, although enriched } \\
\text { for cases of double-hit lymphoma and MHG. }\end{array}$} \\
\hline & & & $B C L 2$ & \\
\hline & & & BCL2 translocation & \\
\hline & & & KMT2D & \\
\hline & & & TNFRSF14 & \\
\hline & & & CREBBP & \\
\hline & & & CREBBP2 & \\
\hline \multirow{7}{*}{ 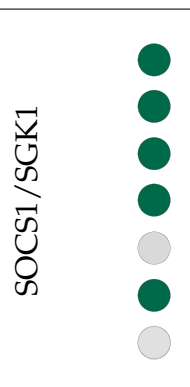 } & \multirow{7}{*}{ Ü } & & CD83 & \multirow{7}{*}{$\begin{array}{l}\text { Predominantly GCB. Shares genetic and gene } \\
\text { expression features of PMBCL. Associated with } \\
\text { the most favorable prognosis. }\end{array}$} \\
\hline & & & HIST1H1E & \\
\hline & & & SGK1 & \\
\hline & & $C$ & NFKBIA & \\
\hline & & & NFKBIE & \\
\hline & & & SOCS1 & \\
\hline & & & $B R A F$ & \\
\hline \multirow{5}{*}{ 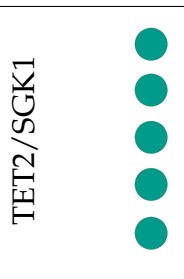 } & & & TET2 & \multirow{5}{*}{$\begin{array}{l}\text { A less strongly identifiable subtype. Has very } \\
\text { strong similarity to SOCS1/SGK1 but differs by } \\
\text { the addition of TET2 and BRAF and the lack of } \\
\text { SOCS1 and CD83. Favorable prognosis. }\end{array}$} \\
\hline & & 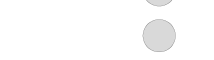 & $B R A F$ & \\
\hline & & 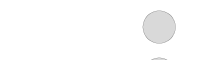 & SGK1 & \\
\hline & &  & KLHL6 & \\
\hline & & 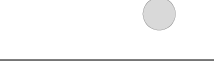 & ID3 & \\
\hline \multirow{8}{*}{ 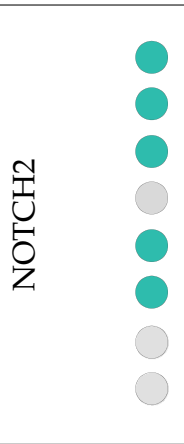 } & \multirow{8}{*}{$\bar{u}$} & \multirow{8}{*}{ 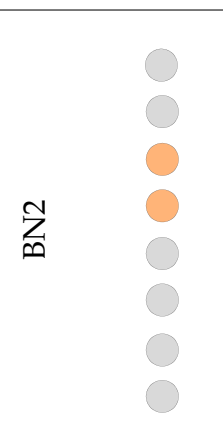 } & BCL10 & \multirow{8}{*}{$\begin{array}{l}\text { Not associated with any COO. Shares mutational } \\
\text { similarity to MZL but not enriched for } \\
\text { transformed MZLs. Less strongly defined than } \\
\text { other subgroups. }\end{array}$} \\
\hline & & & TNFAIP3 & \\
\hline & & & NOTCH2 & \\
\hline & & & BCL6 translocation & \\
\hline & & & CCND3 & \\
\hline & & & SPEN & \\
\hline & & & $U B E 2 A$ & \\
\hline & & & CD70 & \\
\hline \multirow{5}{*}{$\bigcup_{\text {YII }}^{\cup}$} & 0 & \multirow{5}{*}{$\frac{\dot{\Xi}}{\tilde{\Xi}}$} & NOTCH1 & \multirow{3}{*}{$\begin{array}{l}\text { A default category, containing cases that could not } \\
\text { be classified elsewhere and no detected mutation. } \\
\text { Likely to also contain cases belonging to both } \\
\text { NOTCH1 and TP53/CNA subgroups. }\end{array}$} \\
\hline & 0 & & REL amplification & \\
\hline & O & & TP53 & \\
\hline & \multirow{2}{*}{$U$} & & TP53 & \multirow{2}{*}{$\begin{array}{l}\text { Characterized by TP53 mutation and widespread } \\
\text { copy number changes. }\end{array}$} \\
\hline & & & Frequent deletions & \\
\hline
\end{tabular}


Table 2. Cont

\begin{tabular}{cllll}
\hline Lacy et al. & Chapuy et al. & Schmitz et al. & Notes \\
\hline & 8 & $\begin{array}{l}\text { No detected } \\
\text { abnormalities }\end{array}$ & $\begin{array}{l}\text { Cases with no detectable mutation were allocated } \\
\text { to the NEC group. }\end{array}$ \\
\hline & $\mathrm{N} 1$ & NOTCH1 & $\begin{array}{l}\text { Characterized by NOTCH1 mutation, this was } \\
\text { significantly elevated in Lacy's NEC group but } \\
\text { only mutated in 2.5\% of samples. Associated with } \\
\text { poor outcome. }\end{array}$ \\
\hline
\end{tabular}

PCNSL, primary central nervous system lymphoma; FL, follicular lymphoma; MHG, molecular high grade; MZL, marginal zone lymphoma.

Table 3. Implications of genetic subtypes of DLBCL for therapy (from Wright et al., modified).

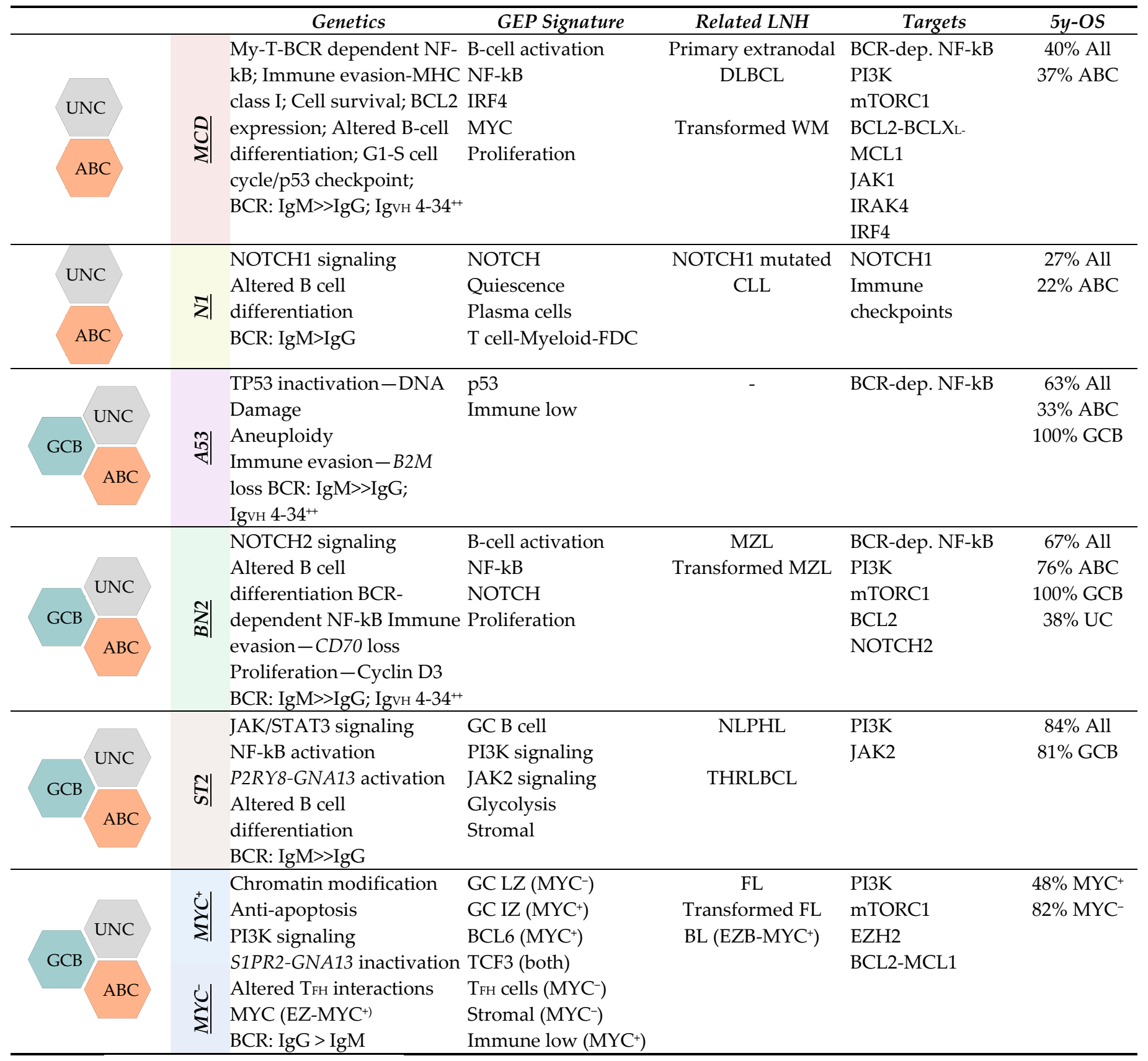

WM, Waldenström macroglobulinaemia; CLL, chronic lymphocytic leukemia; MZL, marginal zone lymphoma; NLPHL, nodular lymphocyte-predominant Hodgkin lymphoma; THRLBCL, T cell histiocyte-. 


\section{Open Issues and Perspectives}

The paper of Lacy et al. [54] was accompanied by a commentary from Morin and Scott [57], who concluded that comprehensive sequencing of a larger number of tumors with the combination of whole-genome and transcriptome sequencing is warranted to develop a new molecular taxonomy which may be concretely translated into clinical benefits. In fact, between $7.5 \%$ and $55 \%$ of the cases reported by Chapuy et al., Schmitz et al., and Lacy et al. did not fit into any of the major genetic categories they identified $[51,53,58]$. The fact that the genomic studies hitherto reported show a certain variability in terms of results may depend on different factors, such as the size of the analyzed cohort or heterogeneity of the techniques used (e.g., FF vs. FFPE tissue, whole exome vs. targeted sequencing, and the statistical approach applied), but also on the actual heterogeneity of the lesions occurring in these tumors. For instance, divergent evolution within the same biopsy, which corresponded to different morphologic, phenotypic, and $\mathrm{COO}$ features [59], has been reported. Although the distinct components had a common clonal origin and shared the bulk of genetic aberrations, each revealed private mutations, in keeping with the above-mentioned morpho-phenotypic and molecular differences.

The heterogeneity of genetic lesions is much greater than was thought until a couple of years ago. This has been highlighted by liquid biopsy (LB) [59-61]. By ultradeep sequencing of the cell-free circulating tumoral DNA (cfDNA) released by neoplastic cells undergoing apoptosis, it has been shown that the global mutational landscape of DLBCL is indeed wider than that observed in diagnostic biopsies, which means that different mutations can occur at different anatomic sites. Once a standardized methodology is developed and the cost per test is reduced, $\mathrm{LB}$ can represent a real-time noninvasive tool for disease monitoring. In fact, patients achieving early molecular response (a 2-log decrease of ctDNA after one cycle of standard chemoimmunotherapy) and major molecular response (a 2.5-log decrease after two cycles) show a significantly superior outcome at 24 months independently of IPI and interim positron emission tomography. Conversely, among treatment-resistant subjects, new mutations are acquired in cfDNA, marking resistant clones selected during the clonal evolution.

The continuous development of sequencing and bioinformatic techniques will allow us to achieve the long searched-for goal of using customized therapies based on the molecular characteristics of each individual tumor. Some approaches do appear to be more easily and cheaply applicable to daily life. Nevertheless, the more comprehensive the bioinformatic approach is, the higher the likelihood of overcoming today's standard chemoimmunotherapy and designing chemotherapy-free protocols capable of curing most (if not all) patients, with minimal or no toxic effects.

Funding: The Ministry of Health, Italian Government, Funds R.C. 2021 (to SC and SAP).

Acknowledgments: This review was conducted thanks to the support of the Italian Association for Cancer Research (AIRC 5x1000 grant n. 21198 to SAP) and the Ministry of Health, Italian Government, Funds R.C. 2021 (to SC and SAP).

Conflicts of Interest: The authors declare no conflict of interest.

\section{References}

1. Swerdlow, S.H.; Campo, E.; Harris, N.L.; Jaffe, E.S.; Pileri, S.A.; Stein, H.; Thiele, J. WHO Classification of Tumours of Haematopoietic and Lymphoid Tissues, Revised 4th ed.; International Agency for Research on Cancer: Lyon, France, 2017; p. 2.

2. Harris, N.L.; Jaffe, E.S.; Stein, H.; Banks, P.M.; Chan, J.K.; Cleary, M.L.; Delsol, G.; De Wolf-Peeters, C.; Falini, B.; Gatter, K.C.; et al. A revised European-American classification of lymphoid neoplasms: A proposal from the International Lymphoma Study Group. Blood 1994, 84, 1361-1392. [CrossRef]

3. Jaffe, E.S.; Harris, N.L.; Stein, H.; Vardiman, J. Pathology and Genetics of Tumours of Haematopoietic and Lymphoid Tissues; IARC Press: Lyon, France, 2001.

4. Swerdlow, S.H.; Campo, E.; Harris, N.L.; Jaffe, E.S.; Pileri, S.A.; Stein, H.; Thiele, J.; Vardiman, J.W. WHO Classification of Tumours of Haematopoietic and Lymphoid Tissues, 4th ed.; International Agency for Research on Cancer: Lyon, France, 2008. 
5. Fisher, R.I.; Gaynor, E.R.; Dahlberg, S.; Oken, M.M.; Grogan, T.M.; Mize, E.M.; Glick, J.H.; Coltman, C.A.; Miller, T.P. Comparison of a standard regimen (CHOP) with three intensive chemotherapy regimens for advanced non-Hodgkin's lymphoma. N. Engl. J. Med. 1993, 328, 1002-1006. [CrossRef]

6. Harrysson, S.; Eloranta, S.; Ekberg, S.; Enblad, G.; Jerkeman, M.; Wahlin, B.E.; Andersson, P.-O.; Smedby, K.E. Incidence of relapsed/refractory diffuse large B-cell lymphoma (DLBCL) including CNS relapse in a population-based cohort of 4243 patients in Sweden. Blood Cancer J. 2021, 11, 9. [CrossRef] [PubMed]

7. Sehn, L.H.; Salles, G. Diffuse large B-cell lymphoma. N. Engl. J. Med. 2021, 384, 842-858. [CrossRef]

8. Alizadeh, A.A.; Eisen, M.B.; Davis, R.E.; Ma, C.; Lossos, I.S.; Rosenwald, A.; Boldrick, J.C.; Sabet, H.; Tran, T.; Yu, X.; et al. Distinct types of diffuse large B-cell lymphoma identified by gene expression profiling. Nat. Cell Biol. 2000, 403, 503-511. [CrossRef] [PubMed]

9. Rosenwald, A.; Wright, G.; Chan, W.C.; Connors, J.M.; Campo, E.; Fisher, R.I.; Gascoyne, R.D.; Muller-Hermelink, H.K.; Smeland, E.B.; Giltnane, J.M.; et al. The use of molecular profiling to predict survival after chemotherapy for diffuse large-B-cell lymphoma. N. Engl. J. Med. 2002, 346, 1937-1947. [CrossRef] [PubMed]

10. Nyman, H.; Jerkeman, M.; Karjalainen-Lindsberg, M.-L.; Banham, A.H.; Leppä, S. Prognostic impact of activated B-cell focused classification in diffuse large B-cell lymphoma patients treated with R-CHOP. Mod. Pathol. 2009, 22, 1094-1101. [CrossRef]

11. Wright, G.; Tan, B.; Rosenwald, A.; Hurt, E.H.; Wiestner, A.; Staudt, L.M. A gene expression-based method to diagnose clinically distinct subgroups of diffuse large B cell lymphoma. Proc. Natl. Acad. Sci. USA 2003, 100, 9991-9996. [CrossRef]

12. Hans, C.P.; Weisenburger, D.D.; Greiner, T.C.; Gascoyne, R.D.; Delabie, J.; Ott, G.; Müller-Hermelink, H.K.; Campo, E.; Braziel, R.M.; Jaffe, E.S.; et al. Confirmation of the molecular classification of diffuse large B-cell lymphoma by immunohistochemistry using a tissue microarray. Blood 2004, 103, 275-282. [CrossRef]

13. Choi, W.W.L.; Weisenburger, D.D.; Greiner, T.C.; Piris, M.A.; Banham, A.H.; Delabie, J.; Braziel, R.M.; Geng, H.; Iqbal, J.; Lenz, G.; et al. A new immunostain algorithm classifies diffuse large B-cell lymphoma into molecular subtypes with high accuracy. Clin. Cancer Res. 2009, 15, 5494-5502. [CrossRef]

14. Colomo, L.; López-Guillermo, A.; Perales, M.; Rives, S.; Martínez, A.; Bosch, F.; Colomer, D.; Falini, B.; Montserrat, E.; Campo, E.; et al. Clinical impact of the differentiation profile assessed by immunophenotyping in patients with diffuse large B-cell lymphoma. Blood 2003, 101, 78-84. [CrossRef]

15. Muris, J.J.F.; Meijer, C.J.L.M.; Vos, W.; Van Krieken, J.H.J.M.; Jiwa, N.M.; Ossenkoppele, G.J.; Oudejans, J.J. Immunohistochemical profiling based on Bcl-2, CD10 and MUM1 expression improves risk stratification in patients with primary nodal diffuse large B cell lymphoma. J. Pathol. 2006, 208, 714-723. [CrossRef] [PubMed]

16. Meyer, P.N.; Fu, K.; Greiner, T.C.; Smith, L.M.; Delabie, J.; Gascoyne, R.D.; Ott, G.; Rosenwald, A.; Braziel, R.M.; Campo, E.; et al. Immunohistochemical methods for predicting cell of origin and survival in patients with diffuse large B-Cell lymphoma treated with rituximab. J. Clin. Oncol. 2011, 29, 200-207. [CrossRef] [PubMed]

17. Zinzani, P.L.; Dirnhofer, S.; Sabattini, E.; Alinari, L.; Piccaluga, P.P.; Stefoni, V.; Tani, M.; Musuraca, G.; Marchi, E.; Falini, B.; et al. Identification of outcome predictors in diffuse large B-cell lymphoma. Immunohistochemical profiling of homogeneously treated de novo tumors with nodal presentation on tissue micro-arrays. Haematologica 2005, 90, 341-347. [PubMed]

18. Visco, C.; Li, Y.; Xu-Monette, Z.Y.; Miranda, R.N.; Green, T.M.; Li, Y.; Tzankov, A.; Wen, W.; Liu, W.-m.; Kahl, B.S.; et al. Comprehensive gene expression profiling and immunohistochemical studies support application of immunophenotypic algorithm for molecular subtype classification in diffuse large B-cell lymphoma: A report from the International DLBCL Rituximab-CHOP Consortium Program Study. Leukemia 2012, 26, 2103-2113. [CrossRef] [PubMed]

19. de Jong, D.; Rosenwald, A.; Chhanabhai, M.; Gaulard, P.; Klapper, W.; Lee, A.; Sander, B.; Thorns, C.; Campo, E.; Molina, T.; et al. Immunohistochemical prognostic markers in diffuse large B-cell lymphoma: Validation of tissue microarray as a prerequisite for broad clinical applications-A study from the Lunenburg Lymphoma Biomarker Consortium. J. Clin. Oncol. 2007, 25, 805-812. [CrossRef] [PubMed]

20. Gutiérrez-García, G.; Cardesa-Salzmann, T.; Climent, F.; González-Barca, E.; Mercadal, S.; Mate, J.L.; Sancho, J.M.; Arenillas, L.; Serrano, S.; Escoda, L.; et al. Gene-expression profiling and not immunophenotypic algorithms predicts prognosis in patients with diffuse large B-cell lymphoma treated with immunochemotherapy. Blood 2011, 117, 4836-4843. [CrossRef] [PubMed]

21. Scott, D.W.; Wright, G.W.; Williams, P.M.; Lih, C.-J.; Walsh, W.; Jaffe, E.S.; Rosenwald, A.; Campo, E.; Chan, W.C.; Connors, J.M.; et al. Determining cell-of-origin subtypes of diffuse large B-cell lymphoma using gene expression in formalin-fixed paraffin-embedded tissue. Blood 2014, 123, 1214-1217. [CrossRef] [PubMed]

22. Scott, D.W.; Mottok, A.; Ennishi, D.; Wright, G.W.; Farinha, P.; Ben-Neriah, S.; Kridel, R.; Barry, G.S.; Hother, C.; Abrisqueta, P.; et al. Prognostic significance of diffuse large B-cell lymphoma cell of origin determined by digital gene expression in formalin-fixed paraffin-embedded tissue biopsies. J. Clin. Oncol. 2015, 33, 2848-2856. [CrossRef]

23. Jais, J.P.; Molina, T.J.; Ruminy, P.; Gentien, D.; Reyes, C.; Scott, D.W.; Rimsza, L.M.; Wright, G.; Gascoyne, R.D.; Staudt, L.M.; et al. Reliable subtype classification of diffuse large B-cell lymphoma samples from GELA LNH2003 trials using the Lymph2Cx gene expression assay. Haematologica 2017, 102, e404-e406. [CrossRef]

24. Gifford, G.; Gabrielli, S.; Gill, A.; Greenwood, M.; Wong, K.; Best, G.; Nevell, D.; Mcllroy, K.; Kliman, D.; Ilmay-Gillespie, L.; et al. Lymphoma cell-of-origin assignment by gene expression profiling is clinically meaningful across broad laboratory contexts. $\mathrm{Br}$. $\mathrm{J}$. Haematol. 2018, 181, 272-275. [CrossRef] 
25. Derenzini, E.; Mazzara, S.; Melle, F.; Motta, G.; Fabbri, M.; Bruna, R.; Agostinelli, C.; Cesano, A.; Corsini, C.A.; Chen, N.; et al. A 3-gene signature based on MYC, BCL-2 and NFKBIA improves risk stratification in diffuse large B-cell lymphoma. Haematologica 2020. Online ahead of Print. [CrossRef]

26. Staiger, A.M.; Ziepert, M.; Horn, H.; Scott, D.W.; Barth, T.F.E.; Bernd, H.-W.; Feller, A.C.; Klapper, W.; Szczepanowski, M.; Hummel, M.; et al. Clinical impact of the cell-of-origin classification and the MYC/BCL2 dual expresser status in diffuse large B-cell lymphoma treated within prospective clinical trials of the german high-grade non-Hodgkin's lymphoma study group. J. Clin. Oncol. 2017, 35, 2515-2526. [CrossRef]

27. Vitolo, U.; Trněný, M.; Belada, D.; Burke, J.M.; Carella, A.M.; Chua, N.; Abrisqueta, P.; Demeter, J.; Flinn, I.; Hong, X.; et al. Obinutuzumab or rituximab plus cyclophosphamide, doxorubicin, vincristine, and prednisone in previously untreated diffuse large B-cell lymphoma. J. Clin. Oncol. 2017, 35, 3529-3537. [CrossRef]

28. Vitolo, U.; Witzig, T.E.; Gascoyne, R.D.; Scott, D.W.; Zhang, Q.; Jurczak, W.; Özcan, M.; Hong, X.; Zhu, J.; Jin, J.; et al. ROBUST: First report of phase III randomized study of lenalidomide/R-CHOP (R2-CHOP) vs placebo/R-CHOP in previously untreated ABC-type diffuse large B-cell lymphoma. Hematol. Oncol. 2019, 37, 36-37. [CrossRef]

29. Green, T.M.; Young, K.H.; Visco, C.; Xu-Monette, Z.Y.; Orazi, A.; Go, R.S.; Nielsen, O.; Gadeberg, O.V.; Mourits-Andersen, T.; Frederiksen, M.; et al. Immunohistochemical double-hit score is a strong predictor of outcome in patients with diffuse large B-cell lymphoma treated with rituximab plus cyclophosphamide, doxorubicin, vincristine, and prednisone. J. Clin. Oncol. 2012, 30, 3460-3467. [CrossRef]

30. Hu, S.; Xu-Monette, Z.Y.; Tzankov, A.; Green, T.; Wu, L.; Balasubramanyam, A.; Liu, W.; Visco, C.; Li, Y.; Miranda, R.N.; et al. MYC/BCL2 protein coexpression contributes to the inferior survival of activated B-cell subtype of diffuse large B-cell lymphoma and demonstrates high-risk gene expression signatures: A report from The International DLBCL Rituximab-CHOP Consortium Program. Blood 2013, 121, 4021-4031. [CrossRef] [PubMed]

31. Ziepert, M.; Lazzi, S.; Santi, R.; Vergoni, F.; Granai, M.; Mancini, V.; Staiger, A.; Horn, H.; Löffler, M.; Pöschel, V.; et al. A 70\% cut-off for MYC protein expression in diffuse large B cell lymphoma identifies a high-risk group of patients. Haematologica 2020, 105, 2667-2670. [CrossRef] [PubMed]

32. Chan, W.C. Using gene expression profiling to move beyond MYC/BCL2 rearrangements in high-grade lymphoma. J. Clin. Oncol. 2019, 37, 175-177. [CrossRef]

33. Sha, C.; Barrans, S.; Cucco, F.; Bentley, M.A.; Care, M.A.; Cummin, T.; Kennedy, H.; Thompson, J.S.; Uddin, R.; Worrillow, L.; et al. Molecular high-grade B-cell lymphoma: Defining a poor-risk group that requires different approaches to therapy. J. Clin. Oncol. 2019, 37, 202-212. [CrossRef] [PubMed]

34. Ennishi, D.; Jiang, A.; Boyle, M.; Collinge, B.; Grande, B.M.; Ben-Neriah, S.; Rushton, C.; Tang, J.; Thomas, N.; Slack, G.W.; et al. Double-hit gene expression signature defines a distinct sub-group of germinal center B-cell-like diffuse large B-cell lymphoma. J. Clin. Oncol. 2019, 37, 190-201. [CrossRef]

35. Jost, P.J.; Ruland, J. Aberrant NF-kappaB signaling in lymphoma: Mechanisms, consequences, and therapeutic implications. Blood 2007, 109, 2700-2707. [CrossRef]

36. Davies, A.; Cummin, T.E.; Barrans, S.; Maishman, T.; Mamot, C.; Novak, U.; Caddy, J.; Stanton, L.; Kazmi-Stokes, S.; McMillan, A.; et al. Gene-expression profiling of bortezomib added to standard chemoimmunotherapy for diffuse large B-cell lymphoma (REMoDL-B): An open label, randomised, phase 3 trial. Lancet Oncol. 2019, 20, 649-662. [CrossRef]

37. Bu, R.; Hussain, A.R.; Al-Obaisi, K.A.S.; Ahmed, M.; Uddin, S.; Al-Kuraya, K.S. Bortezomib inhibits proteasomal degradation of $\mathrm{I} \kappa \mathrm{B} \alpha$ and induces mitochondrial dependent apoptosis in activated B-cell diffuse large B-cell lymphoma. Leuk. Lymphoma 2014, 55, 415-424. [CrossRef] [PubMed]

38. Mottok, A.; Wright, G.; Rosenwald, A.; Ott, G.; Ramsower, C.; Campo, E.; Braziel, R.M.; Delabie, J.; Weisenburger, D.D.; Song, J.Y.; et al. Molecular classification of primary mediastinal large B-cell lymphoma using routinely available tissue specimens. Blood 2018, 132, 2401-2405. [CrossRef] [PubMed]

39. Lenz, G.; Wright, G.; Dave, S.S.; Xiao, W.; Powell, J.; Zhao, H.; Xu, W.; Tan, B.; Goldschmidt, N.; Iqbal, J.; et al. Stromal gene signatures in large-B-cell lymphomas. N. Engl. J. Med. 2008, 359, 2313-2323. [CrossRef] [PubMed]

40. Alizadeh, A.A.; Gentles, A.J.; Alencar, A.J.; Liu, C.L.; Kohrt, H.E.; Houot, R.; Goldstein, M.J.; Zhao, S.; Natkunam, Y.; Advani, R.H.; et al. Prediction of survival in diffuse large B-cell lymphoma based on the expression of 2 genes reflecting tumor and microenvironment. Blood 2011, 118, 1350-1358. [CrossRef] [PubMed]

41. Meyer, P.N.; Fu, K.; Greiner, T.; Smith, L.; Delabie, J.; Gascoyne, R.; Ott, G.; Rosenwald, A.; Braziel, R.; Campo, E.; et al. The stromal cell marker SPARC predicts for survival in patients with diffuse large B-cell lymphoma treated with rituximab. Am. J. Clin. Pathol. 2011, 135, 54-61. [CrossRef]

42. Keane, C.; Gill, D.; Vari, F.; Cross, D.; Griffiths, L.; Gandhi, M. CD4+ tumor infiltrating lymphocytes are prognostic and independent of RIPI in patients with DLBCL receiving R-CHOP chemo-immunotherapy. Am. J. Hematol. 2013, 88, $273-276$. [CrossRef]

43. Abdou, A.G.; Asaad, N.; Kandil, M.; Shabaan, M.; Shams, A. Significance of stromal-1 and stromal-2 signatures and biologic prognostic model in diffuse large B-cell lymphoma. Cancer Biol. Med. 2017, 14, 151-161. [CrossRef]

44. Ciavarella, S.; Vegliante, M.C.; Fabbri, M.; De Summa, S.; Melle, F.; Motta, G.; De Iuliis, V.; Opinto, G.; Enjuanes, A.; Rega, S.; et al. Dissection of DLBCL microenvironment provides a gene expression-based predictor of survival applicable to formalin-fixed paraffin-embedded tissue. Ann. Oncol. 2018, 29, 2363-2370. [CrossRef] 
45. Staiger, A.M.; Altenbuchinger, M.; Ziepert, M.; Kohler, C.; Horn, H.; Huttner, M.; Hüttl, K.S.; Glehr, G.; Klapper, W.; Szczepanoswki, M.; et al. Emed Demonstrator Project; German High Grade Non-Hodgkin's Lymphoma Study Group (DSHNHL): A novel lymphoma-associated macrophage interaction signature (LAMIS) provides robust risk prognostication in diffuse large B-cell lymphoma clinical trial cohorts of the DSHNHL. Leukemia 2020, 34, 543-552. [CrossRef]

46. Tripodo, C.; Zanardi, F.; Iannelli, F.; Mazzara, S.; Vegliante, M.; Morello, G.; Di Napoli, A.; Mangogna, A.; Facchetti, F.; Sangaletti, $\mathrm{S}$; ; et al. A spatially resolved dark-versus light-zone microenvironment signature subdivides germinal center-related aggressive $\mathrm{B}$ cell lymphomas. iScience 2020, 23, 101562. [CrossRef]

47. Ennishi, D.; Takata, K.; Béguelin, W.; Duns, G.; Mottok, A.; Farinha, P.; Bashashati, A.; Saberi, S.; Boyle, M.; Meissner, B.; et al. Molecular and genetic characterization of MHC deficiency identifies EZH2 as therapeutic target for enhancing immune recognition. Cancer Discov. 2019, 9, 546-563. [CrossRef]

48. Kotlov, N.; Bagaev, A.; Revuelta, M.V.; Phillip, J.M.; Cacciapuoti, M.T.; Antysheva, Z.; Svekolkin, V.; Tikhonova, E.; Miheecheva, N.; Kuzkina, N.; et al. Clinical and biological subtypes of B-cell lymphoma revealed by microenvironmental signatures. Cancer Discov 2021. Online ahead of Print. [CrossRef] [PubMed]

49. Reddy, A.; Zhang, J.; Davis, N.S.; Moffitt, A.B.; Love, C.L.; Waldrop, A.; Leppa, S.; Pasanen, A.; Meriranta, L.; KarjalainenLindsberg, M.L.; et al. Genetic and functional drivers of diffuse large B cell lymphoma. Cell 2017, 171, 481-494.e15. [CrossRef] [PubMed]

50. Bolen, C.R.; Klanova, M.; Trneny, M.; Sehn, L.H.; He, J.; Tong, J.; Paulson, J.N.; Kim, E.; Vitolo, U.; Di Rocco, A.; et al. Prognostic impact of somatic mutations in diffuse large B-cell lymphoma and relationship to cell-of-origin: Data from the phase III GOYA study. Haematologica 2019, 105, 2298-2307. [CrossRef] [PubMed]

51. Chapuy, B.; Stewart, C.; Dunford, A.J.; Kim, J.; Kamburov, A.; Redd, R.A.; Lawrence, M.S.; Roemer, M.G.M.; Li, A.J.; Ziepert, M.; et al. Molecular subtypes of diffuse large B cell lymphoma are associated with distinct pathogenic mechanisms and outcomes. Nat. Med. 2018, 24, 679-690. [CrossRef] [PubMed]

52. Schmitz, R.; Wright, G.W.; Huang, D.W.; Johnson, C.A.; Phelan, J.D.; Wang, J.Q.; Roulland, S.; Kasbekar, M.; Young, R.M.; Shaffer, A.L.; et al. Genetics and pathogenesis of diffuse large B-cell lymphoma. N. Engl. J. Med. 2018, 378, 1396-1407. [CrossRef]

53. Xu-Monette, Z.Y.; Wu, L.; Visco, C.; Tai, Y.C.; Tzankov, A.; Liu, W.-M.; Montes-Moreno, S.; Dybkaer, K.; Chiu, A.; Orazi, A.; et al. Mutational profile and prognostic significance of TP53 in diffuse large B-cell lymphoma patients treated with R-CHOP: Report from an International DLBCL Rituximab-CHOP Consortium Program Study. Blood 2012, 120, 3986-3996. [CrossRef]

54. Lacy, S.E.; Barrans, S.L.; Beer, P.A.; Painter, D.; Smith, A.G.; Roman, E.; Cooke, S.L.; Ruiz, C.; Glover, P.; Van Hoppe, S.J.L.; et al. Targeted sequencing in DLBCL, molecular subtypes, and outcomes: A Haematological Malignancy Research Network report. Blood 2020, 135, 1759-1771. [CrossRef] [PubMed]

55. Ennishi, D.; Healy, S.; Bashashati, A.; Saberi, S.; Hother, C.; Mottok, A.; Chan, F.C.; Chong, L.; Abraham, L.; Kridel, R.; et al. TMEM30A loss-of-function mutations drive lymphomagenesis and confer therapeutically exploitable vulnerability in B-cell lymphoma. Nat. Med. 2020, 26, 577-588. [CrossRef] [PubMed]

56. Wright, G.W.; Huang, D.W.; Phelan, J.D.; Coulibaly, Z.A.; Roulland, S.; Young, R.M.; Wang, J.Q.; Schmitz, R.; Morin, R.D.; Tang, J.; et al. A probabilistic classification tool for genetic subtypes of diffuse large B cell lymphoma with therapeutic implications. Cancer Cell 2020, 37, 551-568.e14. [CrossRef] [PubMed]

57. Morin, R.D.; Scott, D.W. DLBCL subclassification: Divide and conquer? Blood 2020, 135, 1722-1724. [CrossRef]

58. Tabanelli, V.; Melle, F.; Motta, G.; Mazzara, S.; Fabbri, M.; Corsini, C.; Gerbino, E.; Calleri, A.; Sapienza, M.R.; Abbene, I.; et al. Evolutionary crossroads: Morphological heterogeneity reflects divergent intra-clonal evolution in a case of high-grade B-cell lymphoma. Haematologica 2020, 105, e432-e436. [CrossRef]

59. Scherer, F.; Kurtz, D.M.; Newman, A.M.; Stehr, H.; Craig, A.F.M.; Esfahani, M.S.; Lovejoy, A.F.; Chabon, J.J.; Klass, D.M.; Liu, C.L.; et al. Distinct biological subtypes and patterns of genome evolution in lymphoma revealed by circulating tumor DNA. Sci. Transl. Med. 2016, 8, 364ra155. [CrossRef]

60. Rossi, D.; Diop, F.; Spaccarotella, E.; Monti, S.; Zanni, M.; Rasi, S.; Deambrogi, C.; Spina, V.; Bruscaggin, A.; Favini, C.; et al. Diffuse large B-cell lymphoma genotyping on the liquid biopsy. Blood 2017, 129, 1947-1957. [CrossRef] [PubMed]

61. Kurtz, D.M.; Esfahani, M.S.; Scherer, F.; Soo, J.; Jin, M.C.; Liu, C.L.; Newman, A.M.; Dührsen, U.; Hüttmann, A.; Casasnovas, O.; et al. Dynamic risk profiling using serial tumor biomarkers for personalized outcome prediction. Cell 2019, 178, 699-713.e19. [CrossRef] [PubMed] 